Documentation et bibliothèques

DOCUMENTATION BIBLIOTHEQUES

\title{
La BNQ dans ses nouveaux murs
}

The Bibliothèque nationale du Québec Moves to a New Home

La Biblioteca Nacional de Quebec en sus nuevas instalaciones

\section{Diane Arcouette}

Volume 51, numéro 1, janvier-mars 2005

Bibliothèque nationale du Québec

URI : https://id.erudit.org/iderudit/1030116ar

DOI : https://doi.org/10.7202/1030116ar

Aller au sommaire du numéro

\section{Éditeur(s)}

Association pour l'avancement des sciences et des techniques de la documentation (ASTED)

\section{ISSN}

0315-2340 (imprimé)

2291-8949 (numérique)

Découvrir la revue

Citer cet article

Arcouette, D. (2005). La BNQ dans ses nouveaux murs. Documentation et bibliothèques, 51(1), 21-24. https://doi.org/10.7202/1030116ar
Résumé de l'article

Deux édifices abritent désormais les activités et les services de la Bibliothèque nationale du Québec : le centre de conservation, situé au 2275, rue Holt, à Montréal, lieu de l'acquisition, du traitement et de la conservation d'une partie des collections patrimoniales, et la Grande Bibliothèque, située au 475, boulevard De Maisonneuve Est à Montréal, qui regroupe le personnel et les collections de diffusion ainsi que la direction générale et de nombreux services.
Tous droits réservés (C) Association pour l'avancement des sciences et des techniques de la documentation (ASTED), 2005
Ce document est protégé par la loi sur le droit d'auteur. L'utilisation des services d'Érudit (y compris la reproduction) est assujettie à sa politique d'utilisation que vous pouvez consulter en ligne.

https://apropos.erudit.org/fr/usagers/politique-dutilisation/ 


\section{La BNQ dans ses nouveaux murs}

DiANE ARCOUETTE

Coordonnatrice, entretien technique

Direction de la gestion des immeubles

\section{RÉSUMÉ | ABSTRACTS | RESUMEN}

Deux édifices abritent désormais les activités et les services de la Bibliothèque nationale du Québec: le centre de conservation, situé au 2275, rue Holt, à Montréal, lieu de l'acquisition, du traitement et de la conservation d'une partie des collections patrimoniales, et la Grande Bibliothèque, située au 475, boulevard De Maisonneuve Est à Montréal, qui regroupe le personnel et les collections de diffusion ainsi que la direction générale et de nombreux services.

\section{The Bibliothèque nationale du Québec} Moves to a New Home

The activities and services of the Bibliothèque nationale $d u$ Québec are located in two buildings. The conservation centre, located at 2275 Holt Street, houses the activities related to the acquisition, cataloguing and conservation of part of the heritage collections. Located at 475 De Maisonneuve Boulevard East, the Grande Bibliothèque is home to the staff and general collections, as well as to management and several other services.

\section{La Biblioteca Nacional de Quebec en sus nuevas instalaciones}

Las actividades y los servicios de la Biblioteca nacional de Quebec tienen lugar en dos edificios: el centro de conservación, situado en la calle Holt 2275, Montreal, lugar donde se hacen las adquisiciones, la catalogación y la conservación de una parte de las colecciones patrimoniales, y la Biblioteca Grande, situada en el bulevar de Maisonneuve Est 475, Montreal, donde se encuentra el personal y las colecciones de difusión, además de la dirección general y otros muchos servicios.
A Bibliothèque NATIONAle DU QuébeC déploie désormais ses activités dans deux édifices aux caractéristiques architecturales uniques.

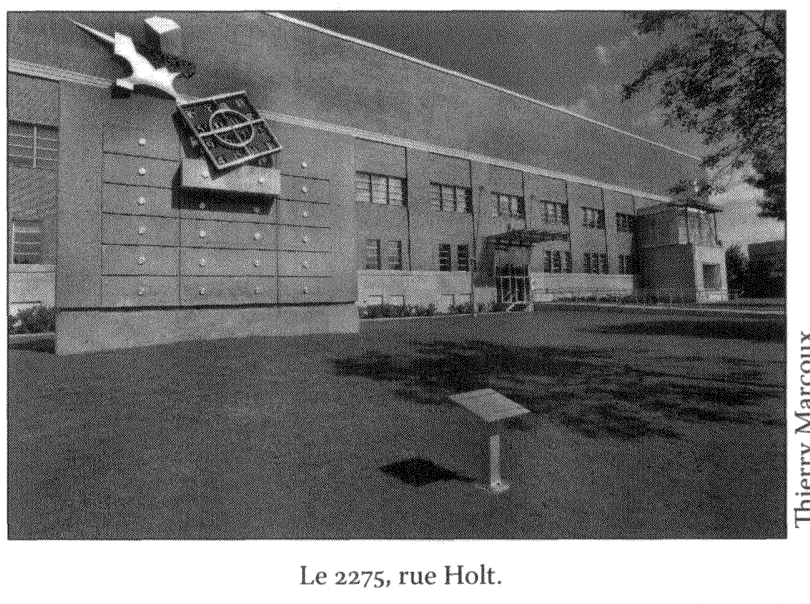

Le 2275, rue Holt, qui abrite, sur 13 ooo $\mathrm{m}^{2}$, l'ensemble des activités liées à la conservation, est un ancien bâtiment industriel de la General Cigar Co construit en 1948 .

À l'origine, l'édifice était doté de spécificités qui devaient se révéler fort intéressantes au regard des missions de la BNQ: excellente circulation de l'air ambiant, filtré et humidifié afin de conserver les feuilles de tabac dans des conditions atmosphériques contrôlées, forte capacité portante des planchers, solidité des charpentes de béton et d'acier. De plus, le bâtiment était chauffé l'hiver et refroidi l'été.

En 1975, l'entreprise de fabrication de cigares déménage rue Saint-Antoine à Montréal, puis à Joliette. L'édifice connaît ensuite plusieurs occupants, notamment la société imprimant les billets vendus par Loto-Québec, puis la Société des loteries de l'Ontario. En raison des impératifs liés à de telles activités, un important dispositif de sécurité est alors installé: les fenêtres sont murées et le terrain est ceint de hautes clôtures métalliques.

Pour devenir le siège social et le centre de conservation de la Bibliothèque nationale du Québec, inauguré en 1997, le bâtiment a dû subir à nouveau d'importants travaux.

Le pari majeur de ce réaménagement consistait à amener la lumière naturelle jusqu'aux aires d'accueil et de travail. Un atrium ouvert sur un puits de lumière est alors mis en place au cœur du bâtiment. L'entrée 


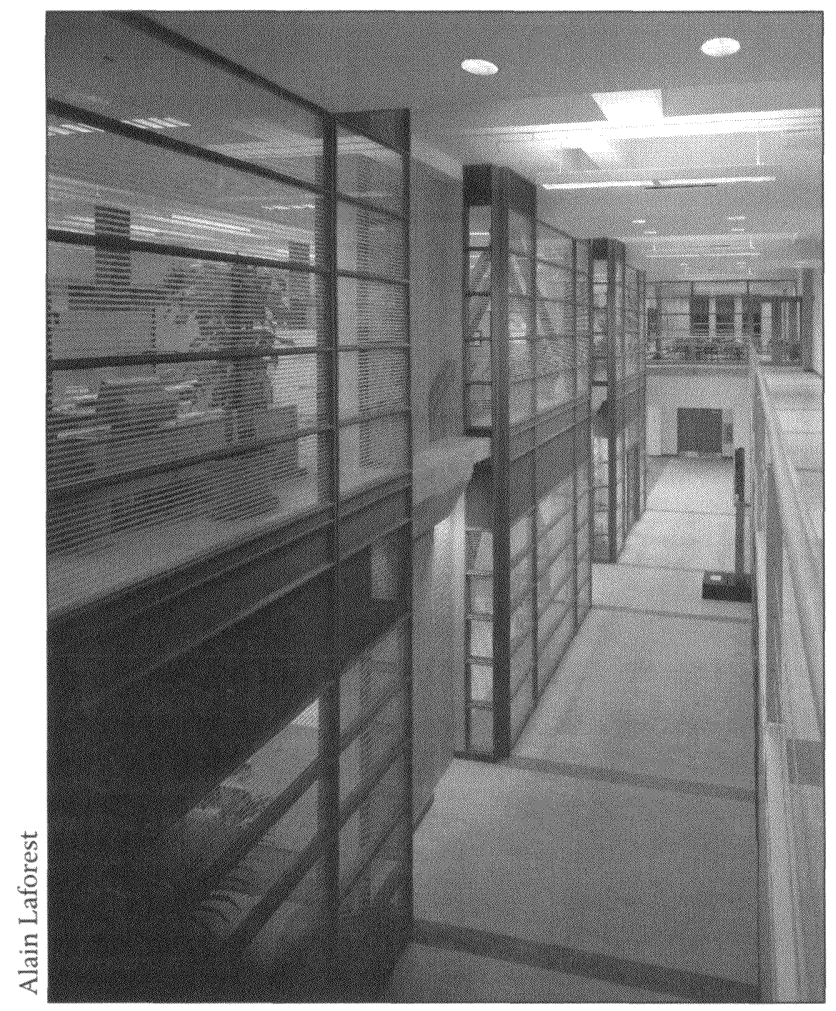

principale est percée au centre de la façade de la rue Holt pour clarifier et simplifier la circulation. Ouverte par de nombreuses fenêtres sur le hall, la salle de lecture réservée aux chercheurs se veut conviviale et fonctionnelle.

Selon leur vocation, les différents espaces ont été réadaptés: entreposage au sous-sol et au troisième niveau du bâtiment; aires publiques au rez-dechaussée; aires de travail des employés au premier étage.

L'extérieur de l'édifice fait, lui aussi, l'objet d'un soin particulier: le revêtement de briques a été décapé et un aménagement paysager a été réalisé aux alentours. Afin de parfaire cette réhabilitation de haut niveau, la BNQ a retenu deux projets dans le cadre du Programme d'intégration des arts à l'architecture: une œuvre sculpturale monumentale de Jacek Jaruszkiewicz installée devant la façade de la rue Holt et une œuvre murale intérieure de Ghislaine Charest exposée dans la salle de repos du personnel.

Avec l'ouverture de la Grande Bibliothèque et le déménagement de plusieurs directions de la BNQ vers ce nouveau lieu, certains espaces de la rue Holt sont de nouveau transformés, notamment pour offrir une plus vaste superficie d'accueil pour les usagers et pour des équipements spécifiques destinés à améliorer leur confort. Au terme de cette restructuration, l'édifice de la rue Holt représente, plus que jamais, un magnifique exemple de reconversion de notre patrimoine industriel.

Quant à la Grande Bibliothèque, située au 475, boulevard De Maisonneuve Est à Montréal, elle permet à la Bibliothèque nationale de développer au mieux sa mission de diffusion et de donner un large accès à d'immenses ressources documentaires. Ce nouveau lieu de culture, implanté au cœur de la ville, est conçu sous le signe de l'ouverture, de la clarté et de la transparence.

À la suite d'un concours international d'architecture, le projet du regroupement d'architectes Patkau/Croft-Pelletier/Gilles Guité a été retenu en 2000 .

Il s'agit d'un bâtiment en aires communicantes de $33000 \mathrm{~m}^{2}$ répartis sur six étages, reliés autour d'un atrium central par trois circulations verticales: des ascenseurs vitrés, des escaliers de terrazzo et une promenade hélicoïdale menant du rez-de-chaussée au troisième étage.

Des atmosphères variées de lecture et de recherche sont créées pour les 2500 places assises réservées aux usagers, dans le confort et la beauté d'un mobilier signé Michel Dallaire.

Les collections sont protégées par deux chambres de bois, situées en périphérie du bâtiment, dont les cloisons ajourées, en bouleau jaune, séparent les espaces de rayonnage des salles de lecture. L'une de ces chambres de bois abrite, sur trois niveaux, la Collection nationale.

Quant à la collection universelle de prêt et de référence, elle déploie ses richesses sur chacun des six niveaux de l'édifice, autour de regroupements thématiques.

Par ailleurs, pour répondre à sa vocation de lieu d'animation culturelle, la Grande Bibliothèque

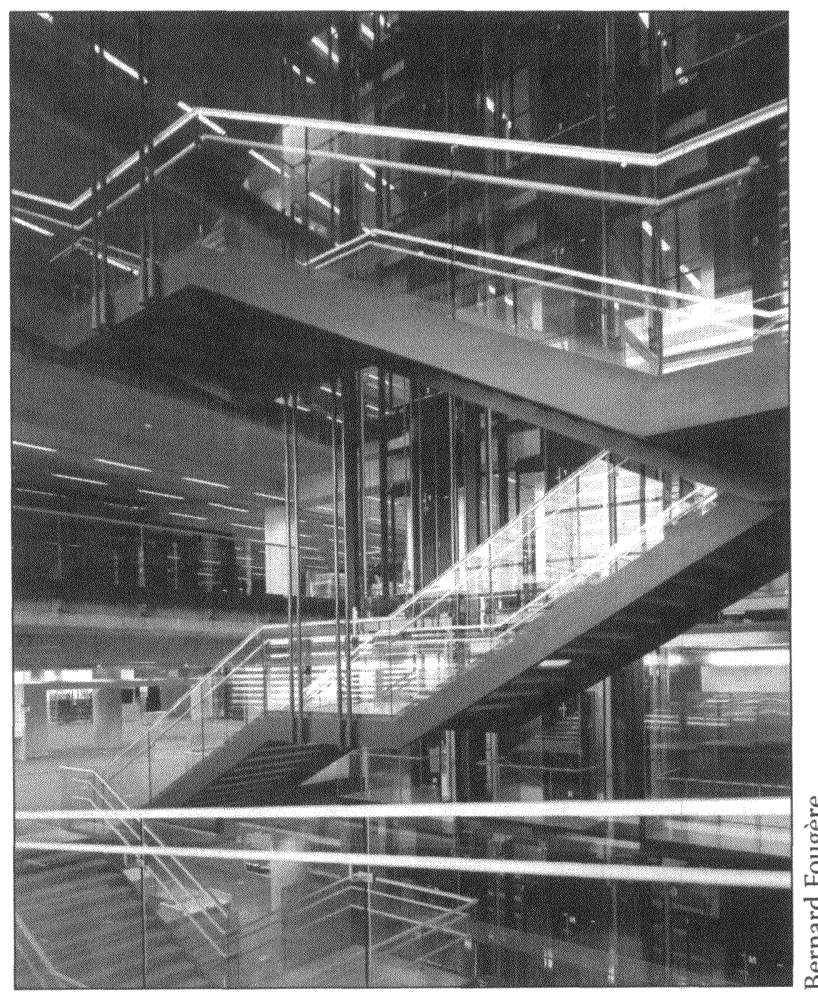




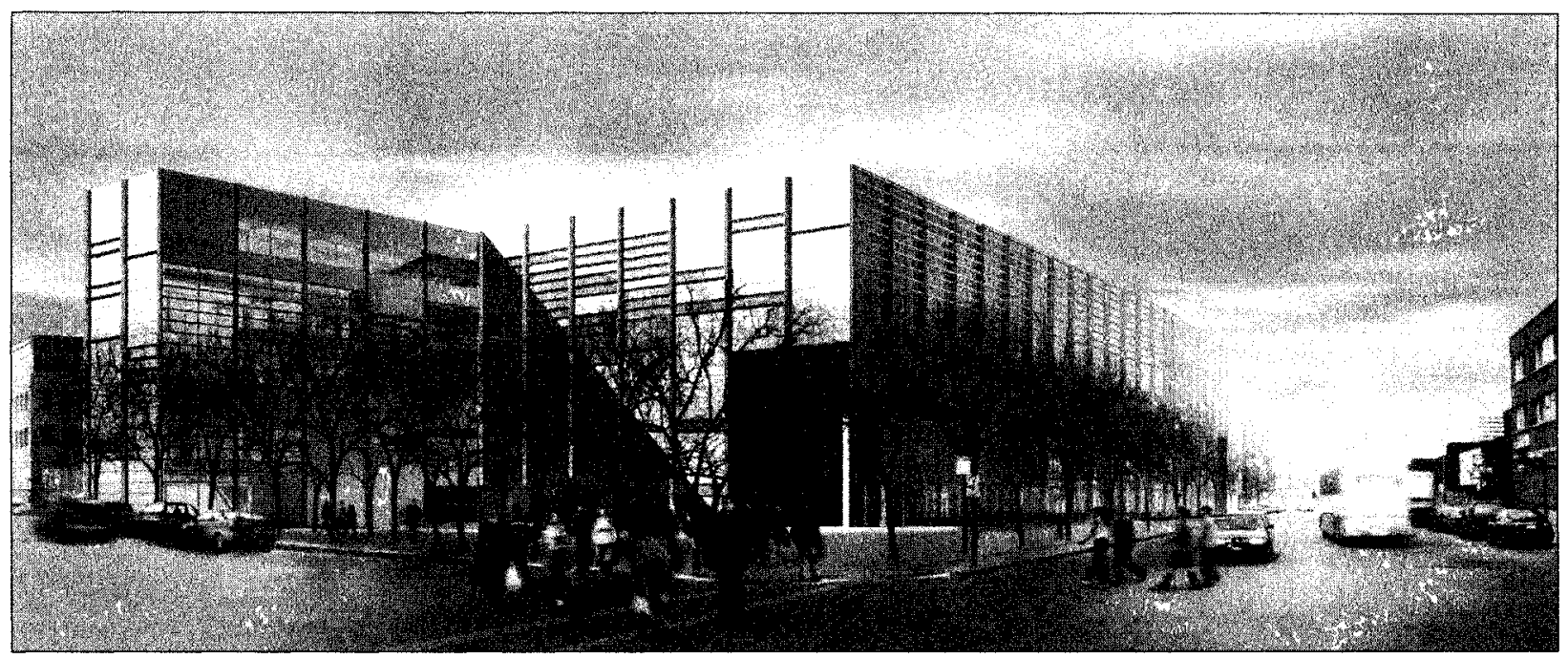

Image de synthèse angle Berri-Maisonneuve: Patkau/Croft-Pelletier/Menkès Shooner Dagenais, architectes associés.

s'est dotée d'un auditorium de 300 places, de salles de conférence et de réunion et de plusieurs espaces d'exposition, dont une salle de $425 \mathrm{~m}^{2}$.

De plus, la construction de la Grande Bibliothèque a bénéficié d'innovations architecturales qui méritent d'être soulignées. Ainsi, toute l'infrastructure de l'édifice est installée dans des planchers surélevés d'environ 60 centimètres de hauteur. Cette solution technologique permet de protéger l'ensemble de ces équipements tout en leur conservant une grande accessibilité. Par ailleurs, première en Amérique du Nord, le revêtement extérieur du bâtiment est composé de 6 ooo lames de verre dépoli, trempées et enduites d'une couche de céramique translucide,

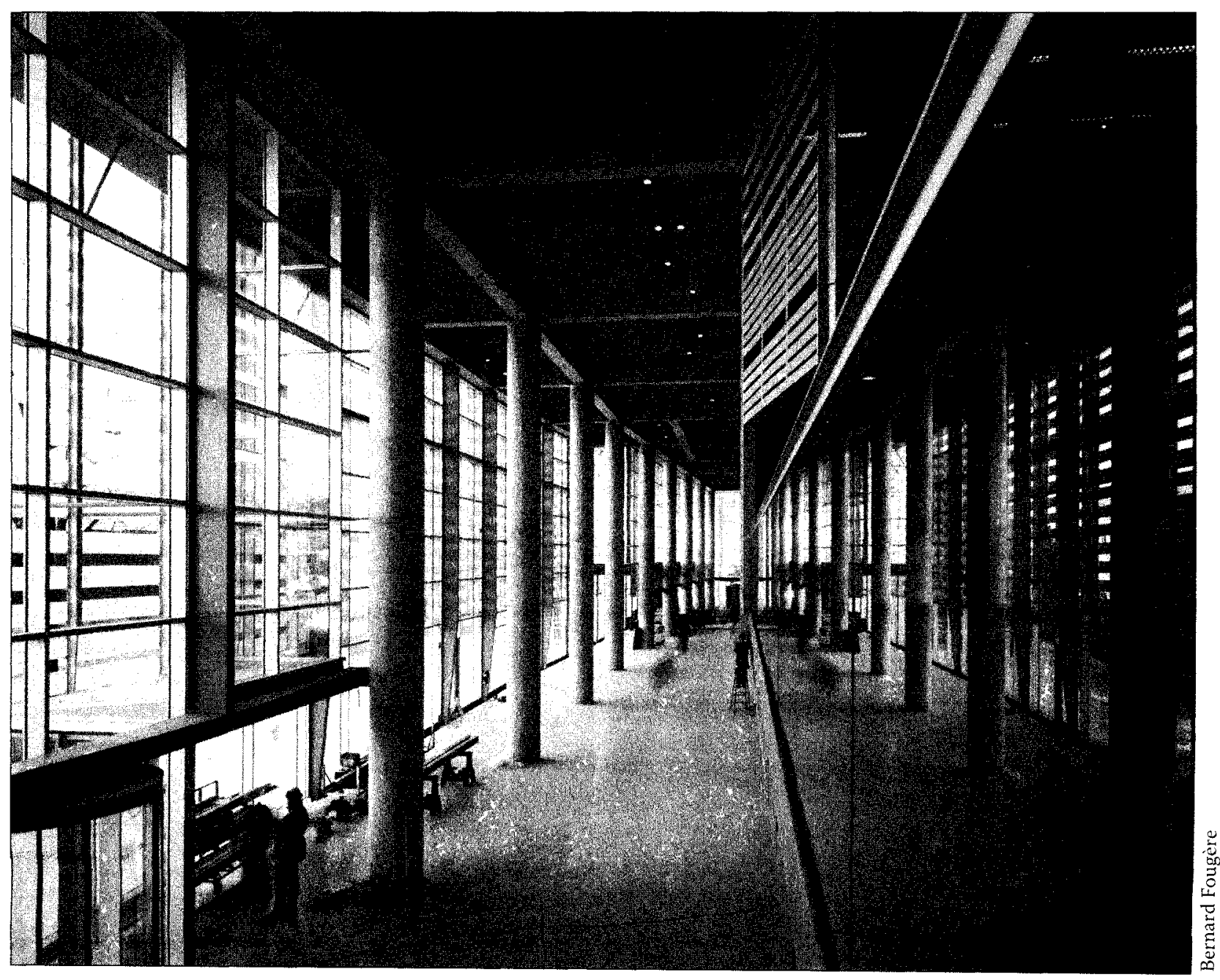




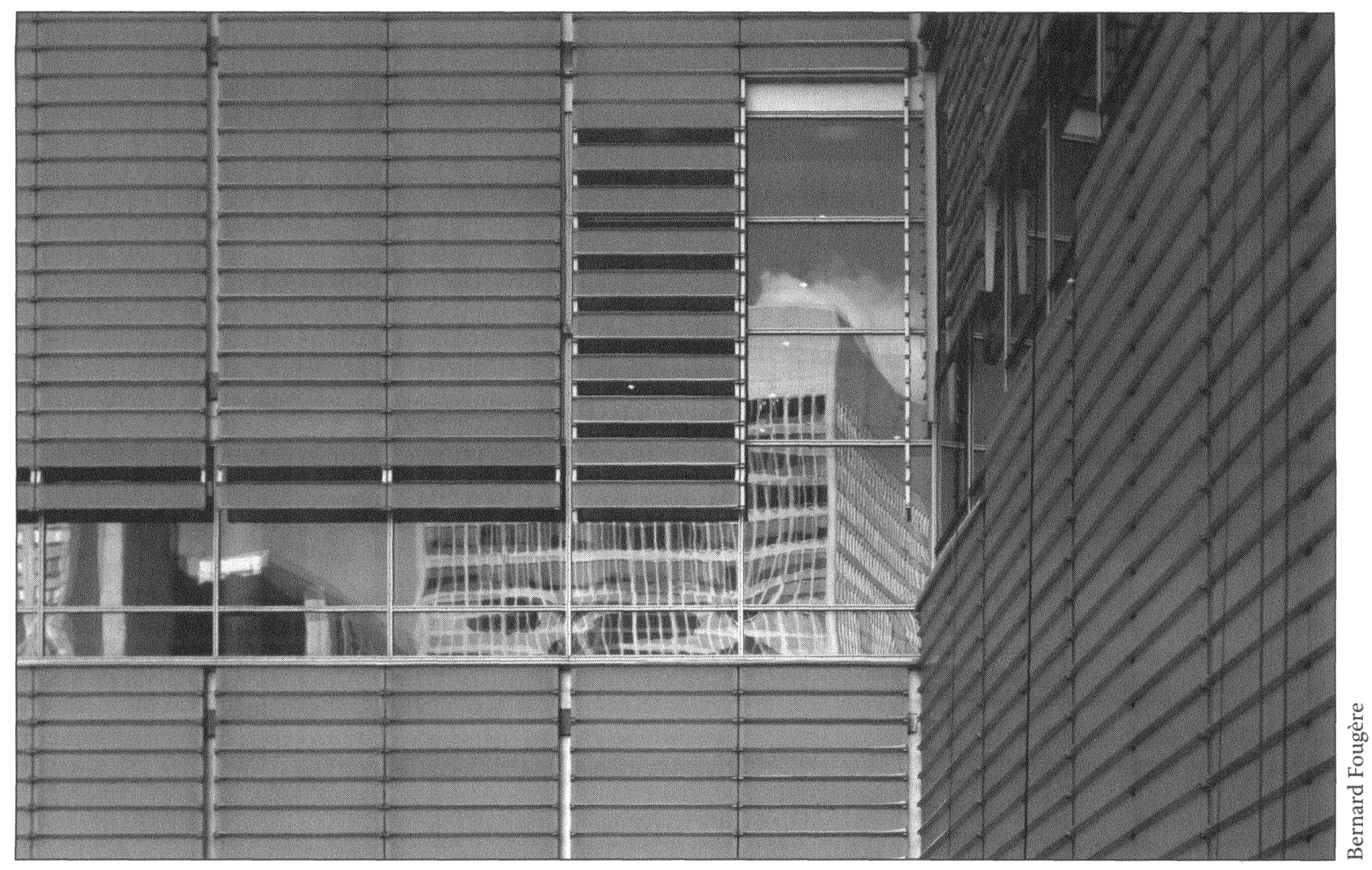

de couleur vert glacier, longues de 2,25 mètres et larges de 35 centimètres. Façonnées dans un four spécialement importé d'Italie, ces lames de verre sont soutenues par une sous-structure d'aluminium et maintenues en place par des griffes d'acier inoxydable. Derrière cet écran, une tôle d'acier galvanisé protège le mur contre les intempéries et complète la chambre d'égalisation des pressions. Cette tôle contribue aussi à réfléchir la lumière naturelle sur le verre qui s'animera ainsi en fonction de l'éclairage naturel.

Il est à noter que le recours extensif au béton armé d'acier pour la construction de l'édifice a valu à la GB le prix Armatura 2004, qui récompense des projets architecturaux novateurs utilisant ce matériau.

Les espaces extérieurs de la Grande Bibliothèque ont, eux aussi, fait l'objet d'aménagements de haute qualité. En effet, le bâtiment lui-même, n'occupe qu'environ la moitié du terrain appartenant à la $B N Q$, et la superficie vacante est mise en valeur à travers l'application du Programme d'intégration des œuvres d'art à l'architecture. Dans ce cadre, quatre œuvres ont été sélectionnées lors d'un concours lancé par le Ministère de la Culture et des Communications, en mai 2002. Au coin sud-est du site, à l'entrée principale de l'édifice, boulevard De Maisonneuve, est aménagée une cour anglaise dont les versants en pente sont agrémentés de plantations d'arbustes. Cette réalisation permet de laisser pénétrer la lumière du jour à l'intérieur du métro et sur le lien de circulation entre le métro et l'édifice. À cet endroit s'élève une sculpture monumentale de Jean-Pierre Morin en acier et en aluminium. Le long de l'avenue Savoie, face à la place Paul-Émile-Borduas, l'une des entrées latérales de lédifice est signalée au public par une ouvre de Dominique Blain intitulée Vous êtes $i c i$. Une troisième œuvre d'art réalisée par Louise Viger, composée de verre, de métal et de lumière est présente dans le couloir menant du métro à la salle d'exposition et au centre de conférences de la Grande Bibliothèque.

Quant à la façade nord de l'édifice, elle s'ouvre sur une vaste esplanade se prolongeant par la quatrième œuvre d'art primée: un jardin cloisonné en 29 lots, dont les deux premiers ont été conçus et agencés par Roger Gauvreau sur les thèmes Jardin punk et Jardin de la forêt urbaine. Chaque année, un nouveau lot sera aménagé par un artiste, l'œuvre étant appelée à évoluer sur plus d'un quart de siècle.

Accueillante, facile d'accès pour les Montréalais et pour l'ensemble des Québécois, grâce à la proximité de la gare centrale d'autobus, la Grande Bibliothèque est destinée à devenir rapidement le lieu d'échange culturel par excellence, adapté aux besoins de notre temps. (-) 